\title{
Current Developments in Wearable Thermometers
}

\author{
Toshiyo Tamura, ${ }^{*, \#}$ Ming Huang, ${ }^{* *}$ Tatsuo Togawa ${ }^{* * *}$
}

\begin{abstract}
Wearable thermometers are popular devices for measuring body temperature during fever, as well as for monitoring basal temperature in women. They are easy to handle, inexpensive, accurate and provide continuous recordings. Most wearable thermometers are connected to a smart phone or tablet to display data. Many types of wearable thermometer are available, such as touch, patch and invisible (radiometric) types. In this review, we describe and discuss currently available wearable thermometers.
\end{abstract}

Keywords: body temperature, wearable thermometer, tattoo thermometer, deep body thermometer, continuous monitoring, thermometer digital, home healthcare.

Adv Biomed Eng. 7: pp. 88-99, 2018.

\section{Introduction}

Temperature, one of the oldest diagnostic parameters, is an important indicator of human health and disease, both in everyday life and in the context of medical care. Measurement of body temperature is easy and accurate. The mercury glass thermometer is popular and shows remarkable reliability. However, safety of the mercury thermometer is inadequate, which has led to the development and popularity of electronic thermometers that use a thermistor. Many other types of thermometer, such as the radiation-type thermometer, have also been developed.

Clinically, fever is a simple index of temperature change. Although change in temperature represents fundamental information, continuous monitoring of body temperature and data processing are inconvenient. Most devices provide only intermittent temperature monitoring.

This review presents various body temperature measurement techniques and discusses currently available wearable devices that monitor body temperature in health and in disease conditions.

\section{Body temperature}

Body temperature can be divided into core and skin sur-

Received on November 21, 2017; revised on March 6, 2018; accepted on March 9, 2018.

* Future Robotics Organization, Waseda University, Tokyo, Japan.

** Nara Institute of Science and Technology, Nara, Japan.

*** School of Human Sciences, Waseda University, Saitama, Japan.

\# Bid 41-5 303, 17 Kikuicho, Shinjuku-ku, Tokyo 162-0044, Japan.

E-mail: tamurat@aoni.waseda.jp face temperature (Fig. 1). Mammals, including humans, are homoeothermic; i.e., they require an almost constant internal body temperature. Core temperature is defined as the temperature of the hypothalamus, which is the regulatory center of the body. Researchers estimate core temperature by taking measurements in the auditory canal, esophagus, and stomach, but rectal temperature is a more accurate method of estimating hypothalamic temperature.

The core temperature is regulated by the thermoregulatory system with its center being the hypothalamus. By means of vasomotor, sweat and evaporation, this system is capable of maintaining the core temperature within a narrow range. Fluctuation of the core temperature obeys the circadian rhythm and shows the lowest value

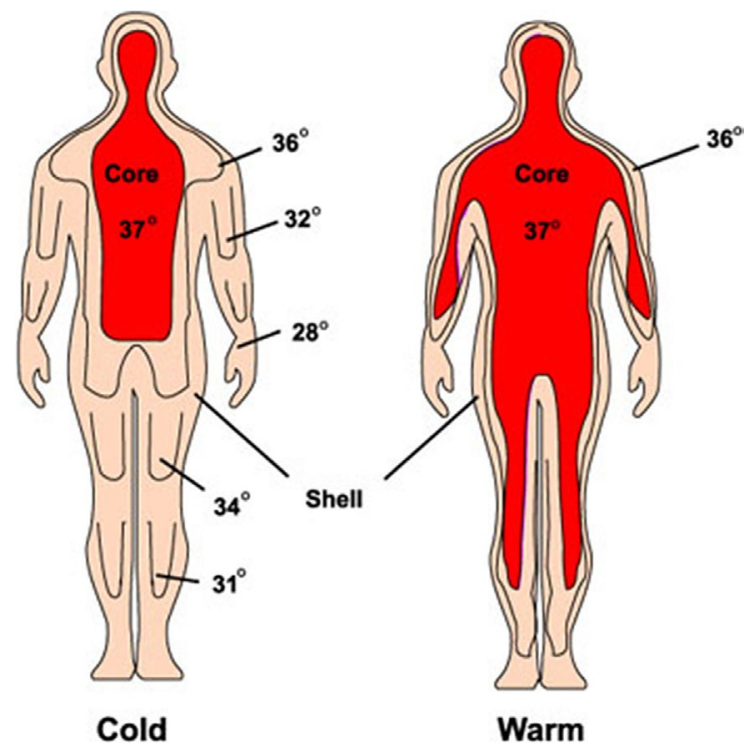

Fig. 1 Core and skin surface temperature distribution. 
in the early morning and a peak in the afternoon or early evening with a difference about $1{ }^{\circ} \mathrm{C}$.

The temperature of the skin of the trunk usually varies between 33.5 and $36.9^{\circ} \mathrm{C}$. The temperature of the skin surface is lower over superficial veins than over superficial arteries. It is also lower over protruding and markedly curved parts such as the nose, ears, fingers and toes.

The advantage of measuring skin temperature is the ease of access for the thermometer. However, the skin is located at the boundary of media with different temperatures. Moreover, fairly marked temperature gradients may develop, both in the tissue and in the environment, due to airflow, thermal radiation, sweating, skin blood flow and coverage by cloth.

Though the skin temperature is heterogeneous and vulnerable to ambient environment, it could still be a plausible index reflecting the change of core temperature according to the site of measurement and the level of activity [1]. The skin temperature correlates to the core temperature by the thermoregulatory system as the main path to conduct heat exchange with the environment. Hence, under thermoneutral situation, the skin temperature on the sites, e.g. the upper thorax or the axilla, with relatively less influence by ambient environment, may be capable to reflect the change of core temperature [2]. These phenomena may be assumed the core temperature from the skin temperature.

\section{Intermittent and continuous temperature monitoring}

Most temperature monitoring is intermittent. In general monitoring applications such as checking for fever, intermittent temperature monitoring using contact and radiation thermometers is common. A probe in contact with the skin surface allows continuous temperature monitoring. Radiation thermometers facilitate non-contact measurement of skin temperature. The emissivity of the surface of the object should be considered to enhance measurement accuracy.

Sites of temperature monitoring, whether continuous or intermittent, are dependent on the patient and their clinical condition. In surgical procedures, short and minimally invasive procedures may only require intermittent measurement, whereas a patient under general anesthesia requires continuous temperature monitoring.

The method most frequently used to monitor temperature may not accurately reflect core temperature, which is the most important determinant of the thermoregulatory response. Sites of temperature monitoring include the skin axilla), esophagus, nasopharynx, tympanic membrane, blood, bladder/urine, and rectum. The skin is the most widely accepted site for temperature monitoring but provides unreliable measurements of the core body temperature. Tympanic probes measure brain temperature via the external carotid artery. However, the probe must be placed perfectly on the tympanic membrane. Nasopharyngeal probes that measure core temperature using respiratory gas can cause epistaxis. Rectal and bladder probes measure core body temperature with relatively easy access. However, the response is delayed if the core temperature changes rapidly. Pulmonary arterial catheters also measure the core temperature, but the technique is invasive. Esophageal probes are commonly used to measure core temperature under anesthesia. The probe, which is inserted via the mouth into the distal third of the esophagus, can also be used to monitor the heart beat and sound.

Basal body temperature (BBT) is defined as body temperature under basal conditions at rest; i.e., the lowest temperature of the body during a 24-h period. When metabolism is minimal, minimal heat is produced. Continuous monitoring of body temperature during sleep would allow determination of the true BBT, and female rhythm can be readily evaluated. Temperature shift is a good biological indicator that ovulation has taken place. The waking body temperature is low during menstruation and remains so until ovulation. After ovulation, the temperature increases by $0.8-1.0^{\circ} \mathrm{C}$. An accurate digital thermometer is required to monitor BBT. Rhythmical studies generally involve intermittent use of a digital thermometer.

Continuous measurement of skin temperature at the axilla is as good as episodic axillary temperature measurement [3].

\section{Principle of temperature detection}

Many different kinds of temperature sensor are available for clinical use, typically integrated into surface probes, catheters, and needles. However, only a small number of them are embedded in wearable devices, with little advances in this application.

Temperature detection can be classified into two types: contact and noncontact measurements. Contact thermometers such as thermistors, and integrated circuits (ICs) measure the average temperature between the sensor and the skin surface. In contrast, non-contact sensors such as thermopiles and pyrosensors measure the intensity of thermal radiation received from a known or calculated area on the surface.

\subsection{Direct measurement}

\subsubsection{Thermistors}

A thermistor is a semiconductor-resistive temperature sensor made from sintered oxides of various metals. In the clinical context, negative temperature coefficient (NTC) thermistors are commonly used as they exhibit a 
proportional decrease in resistance with an increase in temperature. Typically, the temperature coefficient of an NCT thermistor is approximately $0.04 / \mathrm{K}$.

The resistivity of a thermistor, $\rho$, at an absolute temperature $T$ is typically expressed as

$$
\rho=\exp \frac{E_{g}}{2 \kappa T}
$$

where $E_{g}$ is the band gap energy of the semiconductor and $\kappa$ is Boltzmann's constant. When the resistance of a thermistor is $R_{0}$ for a temperature $T_{0}$, the resistance at temperature $T$ can be expressed as

$$
R(T)=R_{0} \exp \left(\left[\frac{1}{T}-\frac{1}{T_{0}}\right] \beta\right)
$$

where $\beta=E_{g} / 2 \kappa$ is a constant dependent on the material of the thermistor. The temperature coefficient $\alpha$ of a thermistor is derived from the above expression as:

$$
\alpha=\frac{1}{R} \frac{d R}{d T}=\frac{d}{d T} \frac{\beta}{T}=-\frac{\beta}{T^{2}}
$$

Typically, thermistor temperature coefficients are non-linear over their operating range, which means that the coefficient itself varies somewhat with temperature. A coefficient is highest at the lowest temperature, and decreases as temperature increases. Thermistor measurement circuits must be linearized to cover large temperature ranges. Figure 2 shows a typical linearizing thermistor output. Figures 2(a) and (b) show resistors configured in parallel and in series, respectively, with the thermistor.

Figure 3 shows an S-shaped resistance/temperature curve for a NTC thermistor combined with a resistor in parallel. The best linearization is obtained by laying the inflation in the middle of the operation temperature target. The resistance of the paralleled resistor can then be calculated with minimum error.

\subsubsection{IC thermometers}

The output across a P-N junction for a constant forward-bias current shows linear temperature dependence over a wide temperature range. For a constant current bias, the voltage drop across a silicon $\mathrm{P}-\mathrm{N}$ diode junction shows a temperature coefficient of approximately

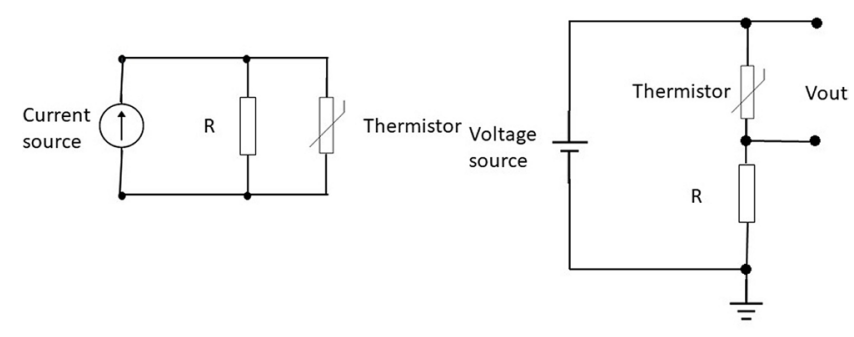

(a)

(b)

Fig. 2 Resistors in parallel (a) and in series (b).
$-2 \mathrm{mV} /{ }^{\circ} \mathrm{C}$. Since the P-N junction is typically used in diodes, transistors, and ICs, temperature sensing can be incorporated into many devices at a low cost. This offers the potential for developing wearable temperature sensors using microprocessors.

\subsection{Indirect measurement}

Infrared radiation thermometers are often used in the clinical setting to measure skin and tympanic temperatures. Humans emit electromagnetic radiation, the power of which correlates precisely with the surface temperature. By determining the radiation intensity, the temperature of an object can be determined without the need for skin contact. In the electromagnetic spectrum, the infrared region spans the wavelength range of 0.8 to $100 \mu \mathrm{m}$, which includes most of the thermal radiation emitted by the human body. The total radiation power, $P_{\text {rad }}$, emitted by an object at temperature $T_{\text {obj }}$ can be expressed as:

$$
P_{\text {rad }}=\sigma \varepsilon T_{\text {obj }}^{4}
$$

where $\sigma$ is the Stefan-Boltzmann constant and $\varepsilon$ is the emission factor of the object. The emission factor lies in the range of 0.85 to 0.95 . This heat-balance equation calculates the net power, $P_{\text {rad }}$, from the sensor readings of two temperatures: $T_{\mathrm{obj}}$ and $T_{\mathrm{amb}}$. Typically, the temperature of the instrument $T_{\mathrm{obj}}$, is equal to the ambient temperature $T_{\text {amb }}$. The total heat power $P_{\text {rad }}$, emitted by the object at temperature, $T_{\mathrm{obj}}$, is shown as:

$$
P_{\text {rad }}=\sigma \varepsilon\left(T_{\mathrm{obj}}^{4}-T_{\mathrm{amb}}^{4}\right)
$$

The sensor generates a voltage $V$, which is proportional to the power of incident radiation $P_{\text {rad }}$.

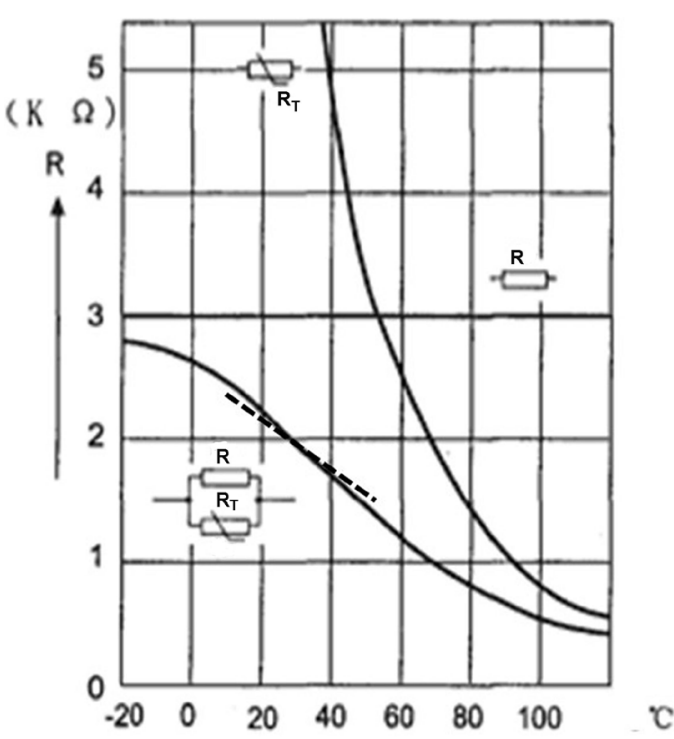

Fig. 3 Resistance/temperature characteristic of a thermistor linearized by a paralleled resistor. $\mathrm{R}$ is a resistance and $\mathrm{R}_{\mathrm{T}}$ is a thermistor. 


$$
V=\mathrm{A} \kappa \varepsilon\left(T_{\mathrm{obj}}^{4}-T_{\mathrm{amb}}^{4}\right)
$$

Here, $\mathrm{A}$ is the amplitude. Typically, the amplitude of the thermocouple output is in the range of microvolts, if the ambient temperature is fixed. The surface temperature of the object can be found from an empirical relation between $V$ and $T_{\text {obj }}$ or from a calibration table. This ambient temperature must be compensated using calibration data to obtain the correct object temperature.

The radiation temperature sensors that are typically used in wearable thermometers are thermopiles or pyroelectric sensors.

\subsubsection{Thermopiles}

The radiation emitted by the object is focused on the receiving element of a suitable thermal-type sensor. This may be a resistance element, which is usually in a thermocouple or thermopile. To measure the emitted radiation power, a thermopile sensor can be used, which consists of a series of thermocouples connected alternately to an active side and a reference side (see Fig. 4). The active side typically consists of a very thin layer of material. The output voltage of this series is proportional to the temperature difference between the active layer and the reference layer. The total radiation is absorbed in the active layer, where a temperature increase proportional to the absorbed radiation power is detected and converted to voltage.

In the thermopile detector, a number of thermocouples are connected in series. Incoming radiation changes the temperature of the thermocouples in the active layer. The measured voltage is obtained by summing the thermocouple voltages in the detector.

The main advantage of a thermopile is stability, as it has the same response to incoming radiant energy regardless of wavelength within the range $0.3-20 \mu \mathrm{m}$. The main disadvantage is that it has a comparatively slow

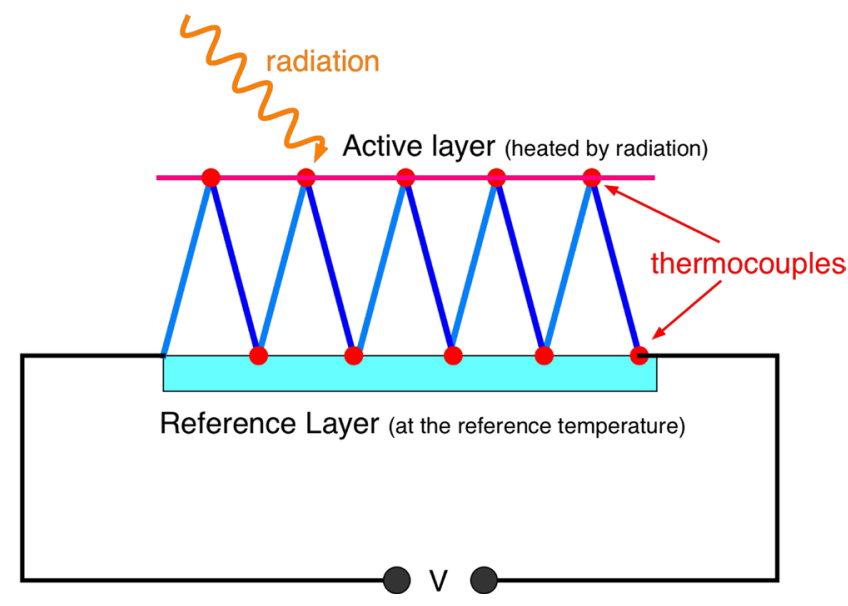

Fig. 4 Schematic of a thermopile detector. speed of response, dependent upon the mass of the thermocouple elements and the rate at which heat is transferred from the hot to cold junctions.

\subsubsection{A Pyroelectric Sensor}

A pyroelectric thermal detector uses a pyroelectric crystal. Temperature fluctuations produce changes in the charge over the surface of pyroelectric crystal, which in turn produces a corresponding electrical signal. This temperature gradient can be created by absorption of the received radiation.

An infrared crystal is used to modulate the power source, using a parallel capacitance housed with additional electronic components. The field effect transducer-based voltage mode has been widely used. Although it has strong temperature-dependent offset, the highest value of $\mathrm{D}^{*}$ can be achieved using a simple circuit. ( $\mathrm{D}^{*}$ represents the signal-to-noise ratio for a particular electrical frequency and bandwidth, if one watt of radiation power reaches a detection surface of $1 \mathrm{~cm}^{2}$.)

Lead zirconate titanate is the material commonly used in consumer products. The incoming radiation must be chopped, and additionally the detector output cannot be used directly. The chopper uses a rotating or oscillating shutter employed to generate AC rather than DC output from the sensor. Relatively weak AC signals are more easily handled by additional conditioning circuitry. The detector change can be likened to a change in charge of a capacitor, which must be read with a high impedance circuit. The AC mode is also preferred when operating at relatively long wavelengths in order to measure low target temperatures, provided by chopping a periodic reference signal proportional to the temperature. An example of an infrared thermometer using a pyroelectric sensor is shown in Fig. 5 [4].

\section{Wearable temperature monitors}

Wearable sensors have undergone substantial development over the past decade, mostly in the context of health-care applications via transduction of physiologi-

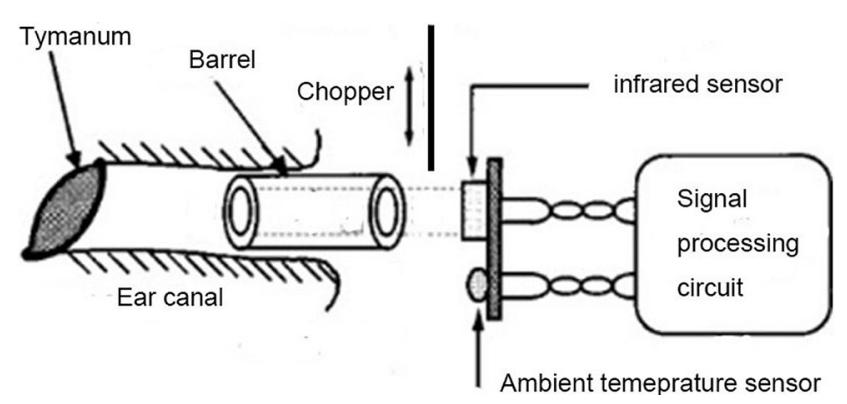

Fig. 5 An example of a pyroelectric thermometer used to measure tympanic temperature. 
cal parameters such as heart rate, respiratory rate, skin temperature and body motion [5-9]. One such commercially available wearable patch shows a temperature differential of $<1.2^{\circ} \mathrm{C}$ compared to a reference device [9].

There has been increasing interest in wearable health-care technology. In this context, the so-called non-invasive wearable health systems for monitoring elderly individuals, patients with chronic diseases, and neonates have been developed. Various approaches have been used for continuous monitoring of vital signs. Temperature monitoring is included because relatively easy constriction of Micro Electro Mechanical Systems works. Moreover, temperature sensors are relatively easy to fabricate.

Wearable and attachable thermometers are popular for monitoring neonates and infants, and for measuring basal temperature. The purpose of such thermometers is monitoring of the skin surface temperature. Simple devices are attached to the head or axilla. In contrast, basal temperature monitoring devices allow continuous recording of temperature at night.

Commercially available thermometers comprise a touchable temperature sensor (either a precision NTC thermistor or a semiconductor sensor), and a Bluetooth wireless transmitter. In most cases, data are collected by a smartphone or tablet. Graphical and tabular views of the data are presented by suitable applications. Temperature is automatically captured daily, and a chart is generated monthly. The client can access the raw data at any time to gain a better understanding of body temperature during periods of sleep. In some devices, data are transferred and securely stored in the cloud.

Numerous wearable touchable thermometers are available commercially (Fig. 6). The major advantage of

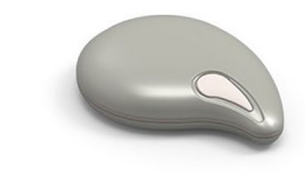

(a) Tempdrop

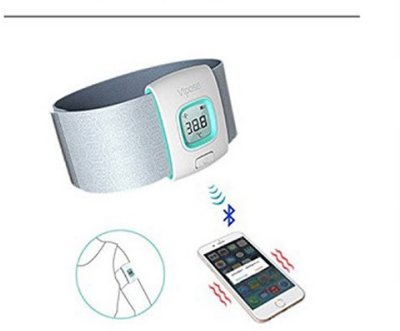

(c) iFiever

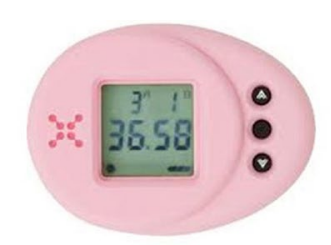

(b) Ran's Night

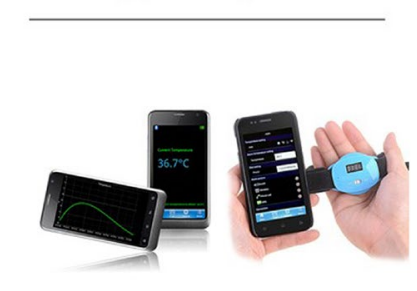

(d) iSense
Fig. 6 Touch-type wearable thermometers. (a) Tempdrop $^{\mathrm{TM}}$, (b) Ran's Night, (c) iFever and (d) iSense. wearable devices is continuous measurement of skin temperature. The daily and monthly rhythms of BBT are related to those of skin temperature, which is measured under cloth or close to the axilla in a constant environment, such as during sleep.

Continuous monitoring of body temperature during the menstrual cycle is commonly performed in daily life. The BBT decreases at the end of the luteal phase. Tempdrop $^{\mathrm{TM}}[10]$ is a smart sensor that turns a smartphone into a sophisticated fertility monitor. The individual simply wears the Tempdrop ${ }^{\mathrm{TM}}$ using an armband or attaching the device directly to the skin while sleeping, and then removes it upon waking. The basal temperature is automatically transferred to a smartphone or tablet. Tempdrop $^{\mathrm{TM}}$, which includes a motion sensor, can evaluate sleep quality, but the accuracy is unclear.

A touchable skin thermometer known as "Ran's Night" $[11,12]$ has been developed and applied for monitoring night-time skin temperature under clothing. The device is $17 \times 41 \times 84 \mathrm{~mm}$ in size, $59 \mathrm{~g}$ in weight and has an accuracy of $\pm 0.5^{\circ} \mathrm{C}$ for a temperature range of 32 $40^{\circ} \mathrm{C}$. The sensor is attached around the abdominal region and measures cutaneous temperature at $10 \mathrm{~min}$ intervals during sleep. The measured temperature data are encoded as a two-dimensional (2D) image and displayed on the LCD of the device. A mobile phone captures the 2D coded images, decodes the information, and transmits the data to a database server.

Monitoring of the body temperature of neonates is also important, but is inconvenient using a traditional thermometer. For this purpose, watch-type thermometers, such as iFever [13] and iSense [14], are available [Fig. 6 (c) and (d)]. The iFever, a smart thermometer comprising an NTC thermistor with an accuracy of $\pm 0.1^{\circ} \mathrm{C}$ at $37.1-42.5^{\circ} \mathrm{C}$, an intelligent LCD monitor and Bluetooth 4.0, can measure temperature at any time by connecting to a smart phone. The skin-friendly and breathable cotton armband that has a length of 14-19 cm is suitable for children aged 0-3 years. The iFever thermometer can operate for $648 \mathrm{~h}$ continuously using a CR2023, 3 V button cell. It includes several functions such as thermometry, temperature alarm, storage of medical records, health management, and cloud backup.

The iSense thermometer is worn using a Velcro bandage. It has an operating range of $35.0-43.0^{\circ} \mathrm{C}$, relative precision of $0.1^{\circ} \mathrm{C}$, and contains a rechargeable battery that allows continuous operation for $40 \mathrm{~h}$.

The BBT in-ear thermometer [15] comprises an earbud thermistor that collects temperature data in the ear canal. The soft silicone material enables the device to fit in the ear canal. Approximately $5 \mathrm{~min}$ is required for the sensor to reach equilibrium at room temperature, while approximately $20 \mathrm{~min}$ is required at lower temperatures. 

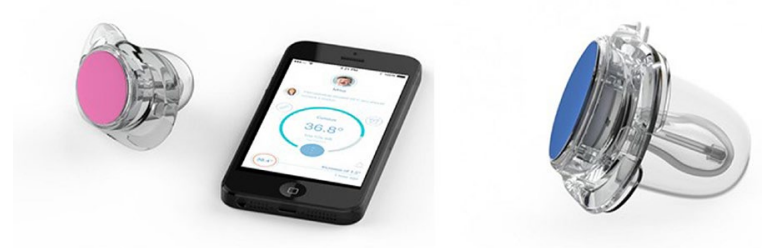

Fig. 7 Digital pacifier thermometer.

The BBT thermometer earbud yields stable temperature readings, and is not affected by the outside temperature. The maximum difference is $0.03^{\circ} \mathrm{C}$ and the average difference is $0.02^{\circ} \mathrm{C}$ compared with a standard thermometer.

For temperature measurements in children, oral, axillary, tympanic (aural), rectal, esophageal, nasopharyngeal, and supralingual (pacifier) routes are used, in addition to via the skin, urinary bladder, pulmonary artery and temporal artery. Numerous studies of temperature measurement in children of various ages have been conducted using a variety of thermometers and routes in both inpatient and outpatient settings.

The Pacif-I [16] (Fig. 7) is a Bluetooth and global positioning satellite (GPS)-enabled pacifier that is linked to a smartphone application. Additionally, it can keep a record of a child's medications, track historical medical data and send this information via email to a pediatrician directly from the application. Pacif-I is made of bisphenol A-free plastic to comply with the safety requirements of the US Consumer Products Safety Commission for pacifiers and is waterproof to facilitate cleaning. The response time is $90 \mathrm{~s}$.

The iButtons ${ }^{\circledR}$ tracking device (Maxim Integrated, San Jose, CA, USA) can also be used for skin temperature measurement in a normal daily environment [17]. The iButtons ${ }^{\circledR}$ device is a digital thermometer that comprises a computer chip enclosed in a $16 \mathrm{~mm}$ stainless steel can. It is sufficiently small and portable to be attached to a ring, watch or other personal item, and reads the temperature of the object to which it is attached. Simply touching a 1 -Wire ${ }^{\circledR}$ probe to the DS1921H reveals the surrounding temperature in the range of $15-46^{\circ} \mathrm{C}$, with an accuracy of $\pm 1{ }^{\circ} \mathrm{C}$ and resolution of $0.125^{\circ} \mathrm{C}$.

In a comparative study with a certified mercury thermometer [18], the temperatures of a temperature-controlled water bath (range: $10-40^{\circ} \mathrm{C}$ ) measured by iButtons ${ }^{\circledR}$ and a thermistor displayed very high correlation with that determined by the reference thermometer $(r>$ 0.999). Prior to correction, the mean bias was $+0.121^{\circ} \mathrm{C}$ for iButtons ${ }^{\circledR}$ and $+0.045^{\circ} \mathrm{C}$ for the thermistor. Upon calibration correction, the mean bias for iButtons ${ }^{\circledR}$ and thermistors was not significantly different from zero. Interestingly, the typical error of the estimate of iButtons ${ }^{\circledR}$ $\left(0.043^{\circ} \mathrm{C}\right)$ was 1.5 -fold less than that of the thermistor $\left(0.062^{\circ} \mathrm{C}\right)$. In a human study, eight healthy males completed three randomized trials at ambient temperatures of 10,20 and $30^{\circ} \mathrm{C}$ while both devices recorded skin temperature at rest (in the presence of low and high wind velocities) and during cycle ergometry exercise. The offset between iButtons ${ }^{\circledR}$ and thermistor readings was generally consistent across conditions, although thermistor readings were always closer to the ambient temperature than those of iButtons ${ }^{\circledR}$, suggesting that the thermistor tended to drift towards environmental conditions. The mean temperature differences between iButtons ${ }^{\circledR}$ and the thermistor during resting trials ranged from $0.261^{\circ} \mathrm{C}$ to $1.356^{\circ} \mathrm{C}$. The mean temperature differences between iButtons ${ }^{\circledR}$ and the thermistor during exercise were $0.989^{\circ} \mathrm{C}$ (ambient temperature, $10^{\circ} \mathrm{C}$ ), $0.415^{\circ} \mathrm{C}$ (ambient temperature, $20^{\circ} \mathrm{C}$ ) and $0.318^{\circ} \mathrm{C}$ (ambient temperature, $30^{\circ} \mathrm{C}$ ). Observed error estimates were within acceptable limits for skin temperature methods, with typical errors $<0.3^{\circ} \mathrm{C}$, correlation coefficients $>0.9$ and cyclic voltammograms $(\mathrm{CVs})<1 \%$ under all conditions. Therefore, use of the wireless iButtons ${ }^{\circledR}$ device is a valid means of measuring human skin temperature during laboratory and field investigations, particularly when skin temperature measurement using other currently available methods may prove problematic.

As mentioned above, the skin temperature fluctuates according to the influences of external and endogenous thermoregulatory systems. However, on the basis of published studies and data using wearable thermometers $[18,19]$, with appropriate installation, the skin temperature reflects the change in core temperature.

\section{Adhesive thermometers}

Three types of adhesive thermometer are available: a small wearable device with an adhesive plaster that is reusable several times, tattoo temperature sensors and disposable sensors. The advantage of an adhesive thermometer lies in its ease of handling and the fact that it can be peeled off easily; its disadvantage is that repeated application and removal of the adhesive material may irritate the skin of neonates.

\subsection{Solid thermometers with adhesive plaster}

Wearable electrical patches were first developed using plastic materials, and consisted of analog front-end electronics and a low-power microprocessor with a built-in radio. The current through the microprocessor varies from a few microamperes with the radio switched off to a few milliamperes with the radio switched on $[8,20$, $21]$. The power usage of the microprocessor is applica- 
tion-dependent. A lithium-ion coin battery with a capacity of several hundred $\mathrm{mAh}$ powers the patch. The electronic patch can be connected wirelessly to a smart phone or tablet via Bluetooth.

Other wirelessly powered patch devices have been developed using thick films [21]. Thick-film electrodes are screen-printed on fabric using planar-fashionable circuit board (P-PCB) technology, in which stainless steel powder is added to reduce both contact impedance and motion artifacts. A programmable gain and bandwidth amplifier is implemented to accommodate various dynamic ranges of vital signals. The sensor must be flexible enough to contact the skin. The flexible printed circuit board can easily monitor vital signs but is too stiff to attach directly to the skin. Thus, a soft adhesive patch is applied between the circuit board and the skin. This type of structure is used in commercially available electrical patches.

Figures 8 (a) and (b) show disposable electrical patches. The FeverFrida ${ }^{\mathrm{TM}}$ thermometer [22] that allows continuous monitoring for up to $48 \mathrm{~h}$ contains a platinum temperature probe in a plastic disk, and is attached under the armpit with adhesive tape. The outside is soft and flexible, including the plastic disk. Data are transmitted at 4-s intervals. The device also alerts the user to fever spikes and reminds the user of the times at which medication is to be administered. The accuracy of the FeverFrida $^{\mathrm{TM}}$ thermometer meets the ASTME1112-00 standard. Feversmart ${ }^{\mathrm{TM}}$ [23] has a similar specification, but no specific details regarding the product are available.

The STEMP sensor [24] that uses medical-grade adhesives works together seamlessly with a smartphone application to provide immediate, accurate and continuous body temperature measurement. The STEMP sensor comprises a rechargeable temperature sensor within a disposable adhesive patch that is attached to the skin under the armpit. It collects data, identifies trends, and

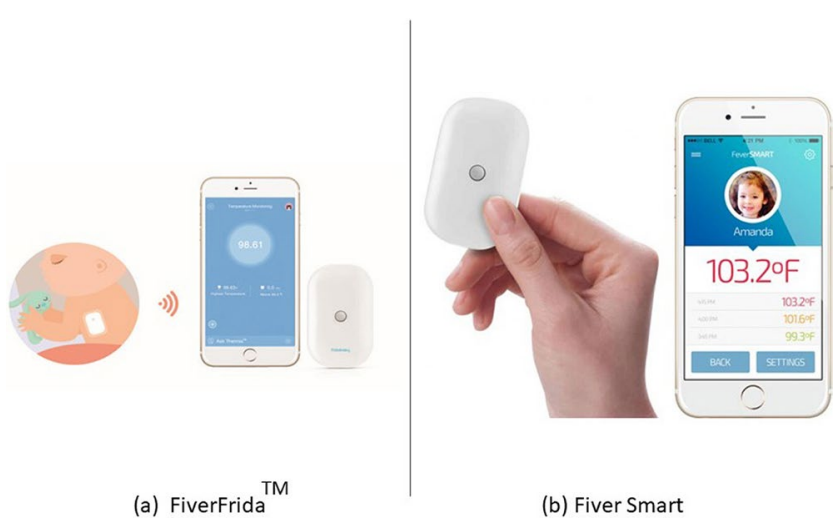

Fig. 8 Patch-type wearable thermometers. (a) FeverFri$\mathrm{da}^{\mathrm{TM}}$ and (b) Fever Smart. makes smart decisions over periods of hours, days, or even weeks. One charge lasts for up to 30 days. The STEMP sensor is accurate to $\pm 0.2^{\circ} \mathrm{C}$. It includes a low-power Bluetooth chip and is housed in a custom-designed, ultrasonically welded plastic case (33 mm long $\times 20 \mathrm{~mm}$ wide $\times 6 \mathrm{~mm}$ thick) that is safe for children and sized appropriately for their smaller bodies (Fig. 9). The integrated lithium polymer (LiPo) battery can be charged rapidly (30 min) using a USB dock, providing up to 30 days of usage on a single charge. The STEMP sensor is factory-sealed to provide a waterproof unit that is safe for use in the shower. The medical-grade adhesive similar to that found in high-quality bandages is safe for children and sensitive skin, even when worn for hours or days (5-7 days is recommended).

\subsection{Temporary transfer tattoo temperature moni- tor}

The novel hybrid fabrication techniques and electronic materials pioneered by Rogers et al. [25-27] have enabled development of advanced electronic devices that can contact the epidermis for measurement of physiological parameters including skin temperature. The sensor circuit is mounted on a biocompatible material, such as

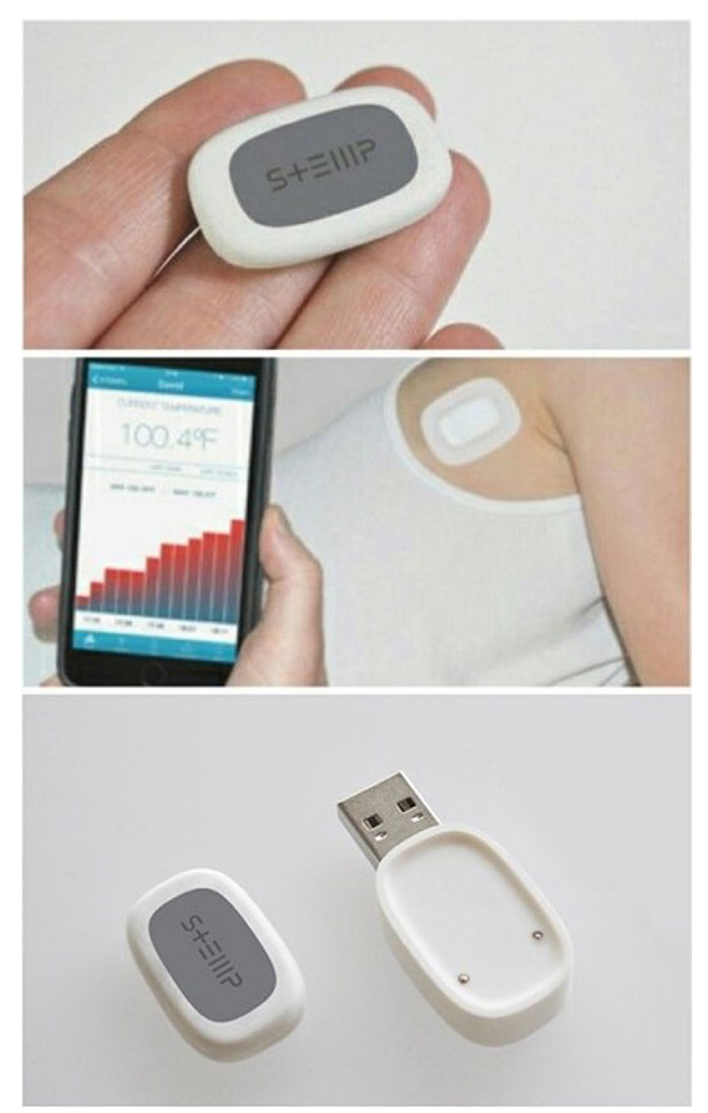

Fig. 9 STEMP sensor. The rechargeable STEMP is inserted into disposable adhesive patch and applied to the body. The STEMP is put on the stand to recharge. 
artificial skin.

Register-based temperature sensors such as platinum resistors are used [25]. These sensors are $50 \mu \mathrm{m}$ wide, or about half the width of the average human hair, and are made of either thin wavy gold wires or silicon membranes mounted on an ultra-thin rubbery sheet that is perforated to help the skin breathe and behave naturally. The sensors adhere to human skin and measure temperatures with millikelvin precision. The prototypes of these devices showed the capability of gathering clinically useful data such as blood flow and skin hydration level with extremely high sensitivity.

An ultrathin, compliant skin-like sensor/actuator can laminate pliably onto the epidermis to provide continuous, accurate thermal measurements. Non-invasive spatial mapping of skin temperature and simultaneous quantitative assessment of tissue thermal conductivity have been demonstrated. Experimental and theoretical studies established the underlying principles of operation, and defined engineering guidelines for device design. Evaluation of subtle variations in skin temperature associated with mental activity, physical stimulation and vasoconstriction/dilation, along with accurate determination of skin hydration through measurements of thermal conductivity represent two important examples of functionality.

Two types of temperature sensor have been produced. The first consists of arrays of temperature sensors that rely on the temperature coefficient of resistance (TCR) in thin (50 nm), narrow $(20 \mathrm{~mm})$ serpentine traces of gold, fabricated using microlithographic techniques and configured for direct external addressing. The second exploits multiplexed arrays of sensors based on PIN diodes formed by patterned doping of $\mathrm{Si}$ nanomembranes.

Changes in temperature cause well-defined shifts in the turn-on voltage. In both cases, the devices can be used as temperature sensors or as local microscale heaters, or as both simultaneously (Fig. 10).

Furthermore, the sensor circuit is mounted on a biocompatible material such as artificial skin, and senses touch, humidity and temperature simultaneously. Monolayer-capped nanoparticle (MCNP) films have high sensitivity for determination of temperature and humidity as well as pressure. The resolution is $1^{\circ} \mathrm{C}$ and the average error is less than 5\% [28].

A commercial disposable and flexible thermometer has also been developed (Fig. 11). Accurate measurement of the body temperature of children and neonates is important, and several wearable wireless thermometers have been developed for this purpose.

Fever Scout [29] is a Bluetooth-enabled, wearable temperature monitor embedded in a soft, comfortable (a)
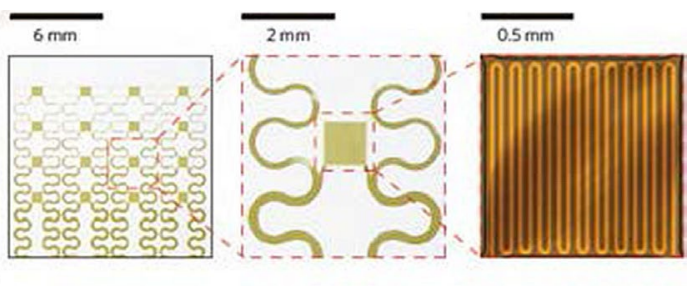

(b)
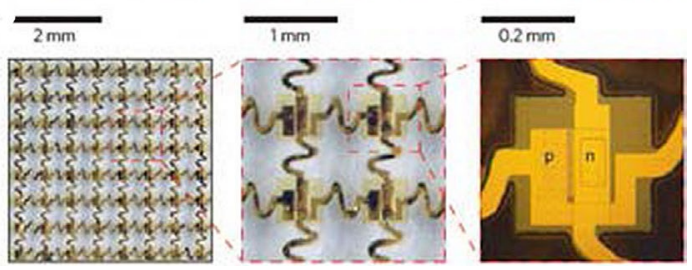

Fig. 10 Two types of epidermal temperature sensors (with permission from Nature Materials [26]. (a) Temperature coefficient of resistance (TCR) sensor, (b) nanomembrane diode sensor.

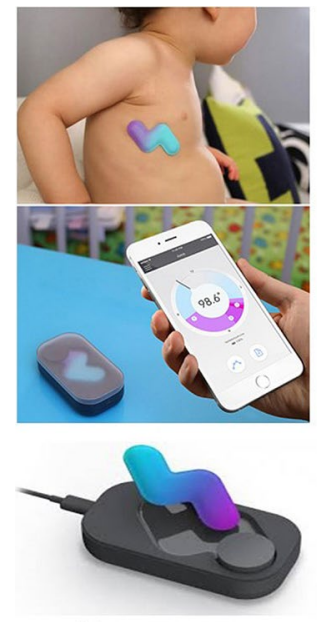

(a) Fever scout
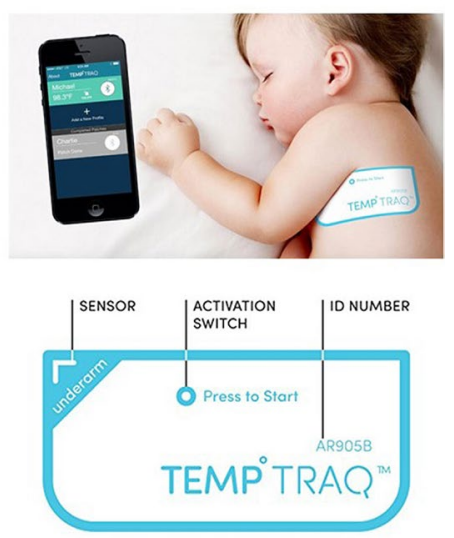

(b) Tempo Traq ${ }^{\mathrm{TM}}$
Fig. 11 (a) Fever Scout (b) TempTraq ${ }^{\mathrm{TM}}$ devices.

patch that provides caregivers with continuous temperature data over $48 \mathrm{~h}$. The thin flexible patch attaches to the underarm skin of the patient. NTC-based wearable thermometers adhere to skin and transmit accurate temperature measurements directly to a smartphone via Bluetooth 4.1.

A thin, soft, breathable sticker (electronic film) with an embedded temperature sensor that comfortably and discreetly adheres to the underarm skin has been developed. The device is $60 \mathrm{~mm}$ in width, $33 \mathrm{~mm}$ in length and $4 \mathrm{~mm}$ in height. The temperature accuracy of $\pm 0.1^{\circ} \mathrm{C}$ meets the ASTM E1112 performance standard. The device is worn for 1-3 days and temperature is read at 1 min intervals. The operation time is $>1$ week per charge of the rechargeable lithium ion battery. The patch is manufactured from silicone and polyurethane.

TempTraq $^{\mathrm{TM}}$ [30] is an intelligent thermometer that 
continuously senses, records and sends alerts of a child's temperature to a smartphone over a 48-h period. TempTraq ${ }^{\mathrm{TM}}$ provides parents and caregivers with the ability to remotely monitor temperature in real-time from any location, through its novel and secure "Connect" service. The Bluetooth-enabled TempTraq ${ }^{\mathrm{TM}}$ is a wearable temperature monitor that takes the form of a soft, comfortable patch.

\section{Radiation thermometer}

Infrared (IR) and microwave radiation can be used in wearable thermometers. A wearable device for skin-contact thermography that uses integrated silicon sensors has been developed $[31,32]$. The static responses of three types of sensor-a thermocouple sensor, passive temperature coefficient temperature sensor and integrated silicon sensor-were simulated, with integrated silicon sensors then being selected. Validation was performed using a commercial oven for the static bench test and a properly designed phantom for the dynamic bench test. The thermal resolution was better than $0.03^{\circ} \mathrm{C}$ and the spatial resolution was $1.6 \mathrm{~m}^{2} \times 10^{-5}$. The authors then investigated its performance in medical application, in which volunteers were subjected to a maximal rate of thermal skin variation equal to $3.1^{\circ} \mathrm{C} / 0.25 \mathrm{~h}$. The results were compared with those obtained using an IR digital video camera. The maximum error was less than $0.14^{\circ} \mathrm{C}$.

An IR camera embedded in a smartphone was used to detect fever [Fig. 12 (a)] [33]. The camera automatically detects the forehead surface, reads the IR camera output signal, calculates the internal body temperature with clinical accuracy and presents the reading on the screen within $1 \mathrm{~s}$. The operating range is 35 to $42^{\circ} \mathrm{C}$ with an accuracy of $0.1^{\circ} \mathrm{C}$, which meets the ISO standard for clinical thermometers.

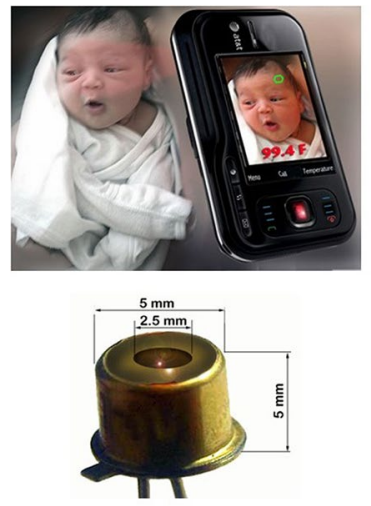

(a)

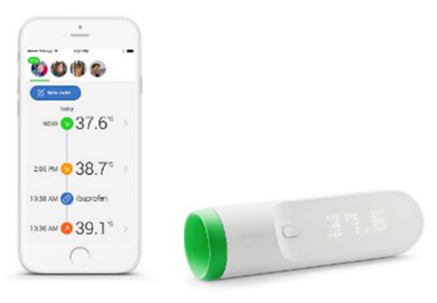

(b)
Fig. 12 Radiation thermometer. (a) Infrared (IR) camera embedded in a smart phone. A pyroelectric sensor is used. (b) A forehead IR sensor.
Microwave radiometry is an attractive method of internal thermometry, and is utilized in a wearable device that can continuously monitor the temperature of different parts of the body, store the data, and transmit it to a digital medical record. A wearable radiometer that operates in two quiet bands (1.4 and $2.7 \mathrm{GHz}$ ) has been developed. The probe, which comprises a flexible substrate, has very low mass and is conformal. Measurements on a three-layer phantom showed the feasibility of internal measurements at depths of several centimeters assuming uniform layers. Relative temperature differences can be measured with an error of less than $0.5 \mathrm{~K}$ and a measurement sensitivity of $0.2 \mathrm{~K}$ [34].

The Withings Thermo [35] uses 16 independent IR sensors to non-invasively measure body temperature using the temporal artery at the side of the head [Fig. 12(b)]. Accordingto Withings, this 'HotSpot Sensor Technology' can rapidly measure IR signature and emitted heat and captures 4,000 measurements in $2 \mathrm{~s}$. A specially designed algorithm corrects for biases such as heat loss and ambient temperature, and then the hottest point corresponded to the temporal artery is determined to yield an accurate single temperature reading. Temperature is detected when the device is placed on the forehead. Clicking a button on the device initiates a temperature measurement, and vibration notifies users of its completion. The LED on the device displays the temperature together with color-coded feedback: green for a normal, orange for a moderate, and red for an elevated temperature. Temperature readings captured by the Thermo are synced automatically to an iOS device over Bluetooth or Wi-Fi and stored in the accompanying application. This enables parents to keep track of trends in their child's temperature over time. Moreover, the data collected can be shared with a physician.

The Thermo device is powered by two AAA batteries, a single set of which can last for up to 2 years. It is denoted as a Class IIa medical device and has been approved by the US Food and Drug Administration.

\section{Wearable deep body thermometers}

Deep body thermometers (DBTs) have been developed to monitor core temperature non-invasively, and were originally proposed in the 1970s by Fox and Solman [36] based on the zero-heat-flow principle. A cutaneous probe is heated to an extent that no temperature gradient exists inside the probe, so that no heat will flow into the probe from the skin and thus no heat will flow from inside the body to the skin. The deep body temperature will then be equal to the value measured by the probe. This method was subsequently improved by Nemoto and Togawa, and Terumo Co. (Tokyo, Japan) [37]. The thermometer fabricated by Terumo has been approved for use as a medical 
device by the Pharmaceuticals and Medical Devices Agency (PMDA) of Japan and has been shown to correlate well with blood temperature during cardiopulmonary surgery [38]. This thermometer uses a heater to compensate for heat flow, and its use as a wearable device is problematic due to insufficient power for longterm use.

To develop a wearable DBT, the dual heat-flux method (DHFM) has been used. DHFM, which is a relatively new method that calculates the DBT based on the heat flux inside a probe, was originally proposed by Kitamura et al. [39]. The double heat path inside the probe enables calculation of the DBT by the temperature sensors. A substrate material with four embedded temperature sensors comprises the core of the probe. The substrate material has physical properties similar to those of skin and, when attached to the skin, most of the heat flow from the core body due to the difference between the DBT and the skin temperature will flow into the substrate material. Additionally, under a heat isolation peripheral boundary condition, heat will flow longitudinally. Since the two heat paths $\left(T_{1}-T_{3}, T_{2}-T_{4}\right)$ are located transversely close to each other, the thermal resistors in the skin layer of the two heat paths are the same, and thus the DBT can be calculated from the measurements of the four sensors ( $\left.T_{1}-T_{4}\right)$ using the equation below, where $k\left(=R_{1} / R_{2}\right)$ is the ratio of heat resistors inside the probes within the two heat paths.

$$
T_{d}=T_{1}+\frac{\left(T_{1}-T_{2}\right)\left(T_{1}-T_{3}\right)}{k\left(T_{2}-T_{4}\right)-\left(T_{1}-T_{2}\right)}
$$

Figure 13(a) shows the location of temperature sensors based on deep body temperature estimation.

A comparative study was performed with reference to a deep body thermometer (CoreTemp, CM-210, Terumo, Tokyo Japan). CoreTemp measures tissue temperature below $10 \mathrm{~mm}$ of skin surface and is used as a stan-

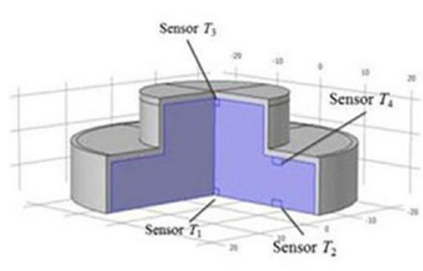

(a)

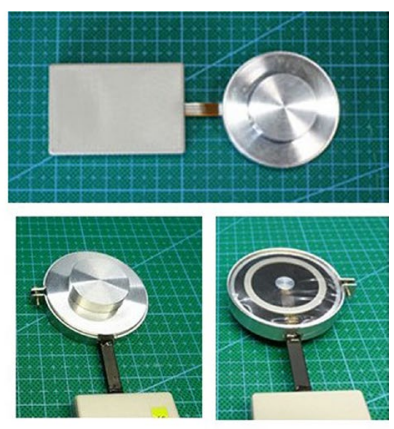

(b)
Fig. 13 Dual heat-flux deep body thermometer monitor. (a) Measurement sites of temperature for dual heatflex deep body thermometer. (b) Prototype of deep body thermometer. dard thermometer for monitoring deep body temperature. The prototype of DHFM device provided measurements with a difference of less than $0.1{ }^{\circ} \mathrm{C}$ compared to the reference thermometer. However, a urethane sponge cover must be used. Huang et al. improved this method by means of theoretical simulation and experimental validation [40-42]. Removal of the external heater markedly reduces the power consumption of the device and thus facilitates its use as a wearable sensor. We have tested the basic performance of this device in practical terms [43] [Fig. 12 (b)].

\section{Conclusion}

Skin temperature is an important physiological measure that reflects the presence of illness and injury as well as provides insight into localized interactions between the body and the environment. Most wearable thermometers were developed for the purpose of continuous monitoring of skin temperature. Most devices meet clinical accuracy standards, such as ISO 80601-2-56 and ASTM E1112. Devices are considered to be in agreement if they meet the clinically meaningful recommendations of mean differences of $\pm 0.5^{\circ} \mathrm{C}$ and limits of agreement of $\pm 1.0^{\circ} \mathrm{C}[44]$. We note that ISO standard and ASTM assume that the clinical accuracy of clinical thermometer can be sufficiently determined under laboratory condition, and tests do not include the characteristics of the patient and the environment. Thus, we evaluate the clinical accuracy in clinical practice.

Although paste-on sensors are inexpensive and allow physicians to monitor patients as they move freely, these devices cannot monitor changes across the skin. Moreover, they irritate the skin, which affects its natural responses. While infrared cameras permit high-precision temperature measurements across the skin, they require patients to maintain a stable posture.

Next-generation temporary tattoo thermometers are promising. In the future, thermometers that can wirelessly broadcast their measurements will likely be developed.

Furthermore, wearable deep body thermometer is promising technology to monitor circadian rhythms and mental stress in daily life. The development of a device with clinically acceptable accuracy will become an issue in the future.

\section{Acknowledgement}

This study was supported by the Keihanna Science City Healthcare Project of the Ministry of Education, Culture, Sports, Science and Technology, Japan, and Health and Labour Sciences Research Grants for Comprehensive Research on Persons with Disabilities, Japan Agency for Medical Research and Development (AMED), 2017- 
2019.

\section{Conflicts of Interest}

The authors declare no conflict of interest.

\section{References}

1. van Someren, Eus JW: Mechanisms and functions of coupling between sleep and temperature rhythms. Prog Brain Res. 153, pp. 309-324, 2006.

2. Scully CG, Karaboué A, Liu W, Meyer J, Innominato P F, Chon $\mathrm{KH}$, Gorbach AM, Lévi F: Skin surface temperature rhythms as potential circadian biomarkers for personalized chronotherapeutics in cancer patients. Interface Focus. 1, pp. 48-60, 2011.

3. van Vliet M, Donnelly JP, Potting CMJ, Blijleven NMA: Continuous non-invasive monitoring of the skin temperature of HSCT recipients. Support Care Cancer. 18, pp. 37-42, 2010.

4. Shibasaki M, Kondo N, Toninaga H, Aoki K, Hasegawa E, Idota Y, Moriwaki T: Continuous measurement of tympanic temperature with a new infrared method using an optical fiber. J Appl Physiol. 85(3), pp. 921-926, 1998.

5. Krause A, Smailagic A, Siewiorek DP: Context-aware mobile computing: learning context-dependent personal preferences from a wearable sensor array IEEE Trans Mobile Comput. 5(2), pp. 113-127, 2006.

6. Windmiller JR, Wang J: Wearable electrochemical sensors and biosensors: a review. Electroanalysis. 2581, pp. 29-46, 2013.

7. Kakria P, Tripathi NK, Kitipawang P: A real-time health monitoring system for remote cardiac patients using smartphone and wearable sensors. Int $\mathrm{J}$ Telemed Appl. 2015, doi. org/10.1155/2015/373474, 2015 .

8. Health patch $\AA$ MD. <http://www.vitalconnect.com/ healthpatch-md> accessed on 3 March, 2018.

9. Selvaraj N: Long-term remote monitoring of vital signs using a wireless patch sensor. 2014 Proceedings of Health Innovations and Point-of-care Technologies Conference. pp. 83-86, 2014.

10. Tempdrop. <http://tempdrop.xyz/?variant=767520619> accessed on 19 February, 2018.

11. Ran's Night Shop.

$<$ http://www.anet21.co.jp/ransnight/index.html> accessed on 19 February, 2018.

12. Chen W, Kitazawa M, Togawa T: Estimation of the biphasic property in a female's menstrual cycle from cutaneous temperature measured during sleep. Ann Biomed Eng. 37(9), pp. 1827$1838,2009$.

13. iFever. $<w w w . v i p o s e . c o m />$ accessed on 19 February, 2018.

14. iSense: smartphone baby temperature sensor and alarm. $<$ http:// www.i-sense.info/> accessed on 3 March, 2018.

15. YONO. The wearable basal thermometer. $<$ https://www.yonolabs.com/product/yono/> accessed on 28 February, 2018.

16. Pacifier Pacif-i. <https://www.pacif-i.io/collections/ frontpage/> accessed on 28 February, 2018.

17. Thermochron iButtons ${ }^{\circledR}$. <https://www.maximintegrated.com/ en/products/ibutton/ibuttons/thermochron.cfm $>$ accessed on 28 February, 2018.

18. Smith ADH, Crabtree DR, Bilzon JLJ, Walsh NP: The validity of wireless iButtons ${ }^{\circledR}$ and thermistors for human skin temperature measurement. Physiol Meas. 31, pp. 95-111, 2010.

19. Zornoza-Moreno M, Fuentes-Hernández S, Sánchez-Solis M, Rol MÁ, Larqué, E, Madrid, AJA. Assessment of circadian rhythms of both skin temperature and motor activity in infants during the first 6 months of life. Chronobiol Int. 28(4), pp. 330337, 2011.

20. Haar RG, Duun S, Thomsen EV, Hoppe K, Branebjerg J: A wearable "electronic patch" for wireless continuous monitoring of chronically deceased patients. Proceedings of the $5^{\text {th }}$ Internal Workshop on Wearable and Implantable Sensor Networks. pp. 66-70, 2008.

21. Yan L, Yoo J, Kim B, Yoo H-J: A $0.5-\mu \mathrm{V}_{\mathrm{rms}} 12-\mu \mathrm{W}$ wirelessly powered patch-type healthcare sensor for wearable body sensor network. IEEE J Solid-state Circuits. 45(11), pp. 2356-2365, 2010.

22. FiverFrida. <http://fridababy.com/product/feverfrida/> accessed on 23 February, 2018.

23. Fiver Smart patch thermometer. <http://feversmart.com/> accessed on 23 February, 2018.

24. STEMP smart temperature patch http://vator.tv/company/ stemp-inc accessed on 23 Feb 2018.

25. Kim DH, Lu N, Ma R, Kim YS, Kim RH, Wang S, Wu J, Won SM, Tao H, Islam A, Yu KJ, Kim T, Chowdhury R, Ying M, Xu L, Li M, Chun HJ, Keum H, McCormick M, Liu P, Zhang YW, Omenetto FG, Huang Y, Coleman T, Rogers JA: Epidermal electronics. Science. 333, pp. 838-843, 2011.

26. Webb RC, Bonifas AP, Behnnaz A, Zhang Y, Yu K-J, Cheng H, Shi M, Bain Z, Liu Z, Kim Y-S, Yeo W-H Park JS, Song J, Li Y, Huang Y, Gorbach AM, John A, Rogers JA: Ultrathin conformal devices for precise and continuous thermal characterization of human skin. Nature Materials. 12, pp 938-944, 2013.

27. Son D, Lee j, Qiao S, Ghaffari R, Kim J, Lee JE, Song C, Kim SJ, Lee DJ, Jun SW, Yang S, Park M, Shin J, Do K, Lee M, Kang K, Hwang CS, Lu N, Hyeon T, Kim D-H: Multifunctional wearable devices for diagnosis and therapy of movement disorder. Nat Nanotechnol. 9: pp. 397-404, 2014.

28. Segev-Bar M, Landman A, Nir-Shaprira M, Shuster G, Haick $\mathrm{H}$ :Tunable touch sensor and combined sensing platform: toward nanoparticle-based electronic skin. ACS Appl Mater Interfaces. 5, pp. 5531-5541, 2013.

29. Fever Scout. <http://www.vivalnk.com/feverscout> accessed on 28 February, 2018.

30. TempTraq ${ }^{\mathrm{TM}}$ wearable patch. $<$ https://www.temptraq.com/> accessed on 28 February, 2018.

31. Giansanti D, Maccioni G, Gigante GE: A comparative study for the development of a thermal odoscope for the wearable dynamic thermography monitoring. Med Eng Phys. 28, pp. 363-371, 2006.

32. Giansanti D, Maccioni G: Development and testing of a wearable integrated thermometer sensor for skin contact thermography. Med Eng Phys. 29, pp. 556-565, 2007.

33. Thermal IR camera for smartphones. <http://www.fraden.com/> accessed on 3 March, 2018.

34. Popovic Z, Momenroodaki P, Scheeler R: Toward wearable wireless thermometers for internal body temperature measurements. IEEE Commun Mag. 52(10), pp. 118-125, 2014.

35. Thermo. <https://health.nokia.com/uk/en/thermo> accessed on 3 March, 2018. 
36. Fox R, Solman A: A new technique for monitoring the deep body temperature in man from the intact skin surface. J Physiol. 212(2), pp 8-10, 1971.

37. Nemoto T, Togawa T: Improved Probe for a Deep Body Thermometer. Med Biol Eng Comput. 26, pp. 456-459, 1988.

38. Yamakage MA, Namiki A: Deep temperature monitoring using a zero-heat-flow method. J Anesth. 17(2), pp. 108-115, 2003.

39. Kitamura K-I, Zhu X, Chen W, Nemoto T: Development of a new method for the noninvasive measurement of deep body temperature without a heater. Med Eng Phys. 32(1), pp. 1-6, 2010.

40. Huang M, Tamura T, Chen W, Kanaya S: Evaluation of structural and thermophysical effects on the measurement accuracy of deep body thermometers based on dual-heat-flux method. $\mathrm{J}$ Therm Biol. 47, pp. 26-31, 2015.

41. Huang M, Tamura T, Tang Z, Chen W, Kanaya S: Structural optimization of a wearable deep body thermometer: From theoretical simulation to experimental verification. J Sens. 2016, Article ID 4828093. dx.doi.org/10.1155/2016/4828093, 2016.

42. Huang M, Tamura T, Chen, N. Ono, Sato T, Kanaya S: Evaluation of a noninvasive deep body thermometer in measurement of specific positions. Conf Proc IEEE Eng Med Biol Soc. pp. 239523982015.

43. Huang M, Tamura T, Tang Z, Chen W, Kanaya S: A wearable thermometry for core body temperature measurement. IEEE J Biomed Health Inf. DOI 10.1109/JBHI.2016.2532933, 2016.

44. Emergency nursing resources development committee: Clinical practice guidelines: non-invasive temperature measurement in the emergency department. pp. 1-14, 2011.

\section{Toshiyo Tamura}

Dr. Toshiyo TAmura received his BS and MS degrees from Keio University, Japan, in 1971 and 73, respectively and Ph.D. from Tokyo Medical and Dental University in 1980 . He is currently a visiting senior researcher, Future Robotics Organization, Waseda University, Japan. His research inter-

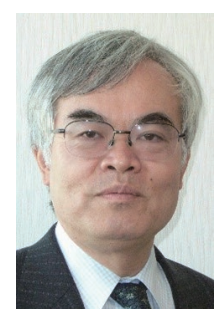
ests include biomedical instrumentation, biosignal processing, telemedicine telecare, home care technology and rehabilitation engineering. His research has resulted in over 120 English reviewed articles. His and his colleagues' book entitled "Biomedical sensors and instruments" is a popular textbook for bio-instrumentation and medical devices.

\section{Ming Huang}

Dr. Ming Huang received his PhD. from the University of Aizu, Japan in 2012. He is currently an assistant professor at Nara Institute of Science and Technology. His research interests include Biomedical engineering and health informatics. He is a member of Engineering in Medicine and Biology, IEEE.

\section{Tatsuo Togawa}

Dr. Tatsuo Togawa received his BS degree from Applied Physics, Waseda University, and MS. Ph.D degrees from Applied Physics, Tokyo University. He served as a Professor in Tokyo Medical and Dental University, and Waseda University. Professional field is biomedical engineering, and human sciences. His recent publication is Biomedical Sensors and Instrumentations by T. Togawa, T. Tamura, P. Å. ÖBerg, CRC Press, 2011.
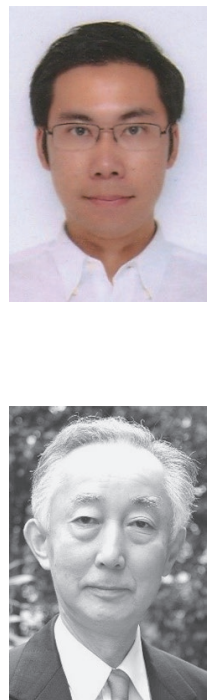